\title{
Autonomic Mechanisms of Emotional Reactivity and Regulation
}

\author{
Catherine C. Uy ${ }^{1}$, Iain A. Jeffrey ${ }^{2}$, Matthew Wilson ${ }^{3}$, Viswanath Aluru ${ }^{1}$, \\ Anita Madan ${ }^{4}$, Ying Lu ${ }^{5}$, Preeti Raghavan ${ }^{1,5^{*}}$ \\ ${ }^{1}$ Department of Rehabilitation Medicine, New York University School of Medicine, New York, USA \\ ${ }^{2}$ Touro College of Osteopathic Medicine, New York, USA \\ ${ }^{3}$ Hunter College, New York, USA \\ ${ }^{4}$ Department of Psychiatry, New York University School of Medicine, New York, USA \\ ${ }^{5}$ Education and Human Development, Steinhardt School of Culture, New York University, New York, USA \\ Email: "Preeti.Raghavan@nyumc.org
}

Received May $15^{\text {th }}, 2013$; revised June $29^{\text {th }}, 2013$; accepted July $23^{\text {rd }}, 2013$

Copyright $(C 2013$ Catherine C. Uy et al. This is an open access article distributed under the Creative Commons Attribution License, which permits unrestricted use, distribution, and reproduction in any medium, provided the original work is properly cited.

\begin{abstract}
The ability to perceive and regulate our emotions appropriately is essential for social behavior. Our subjective emotional states to changing external cues are accompanied by physiological changes in heart rate variability (HRV), which is regulated by the sympathetic and parasympathetic branches of the autonomic nervous systems (ANS). In this pilot study, we sought to elucidate the autonomic basis of emotional reactivity and regulation in response to ecologically-valid emotional stimuli-presented in the form of filmclips - in healthy subjects. Subjects watched a series of videos, validated to elicit feelings of amusement, sexual amusement, sadness, fear, and disgust. Subjects were also asked to regulate the outward expression of their response to disgust by suppressing or amplifying it when instructed. Electrodes placed on the torso measured cardiac and respiratory signals, which were processed to compute HRV, which when analyzed with the concurrent respiratory signal calculates measures of parasympathetic activity (RFA, Respiratory Frequency Area, from higher frequencies) and sympathetic activity (LFA, Low Frequency Area, from lower frequencies). Fluctuations in LFA and RFA were computed by the coefficient of variation, and the intensity of the emotional response to the film-clips was captured via questionnaires. Our results suggest that in healthy individuals, higher intensities of subjective emotional experience, both positive (e.g., amusement) and negative (e.g., amplified disgust) elicit higher LFA (sympathetic) responses, whereas emotional regulation is mediated primarily by fluctuations in RFA (parasympathetic) activity. Furthermore, correlations between emotional intensity and components of HRV suggest that higher positive or lower negative emotional states may increase the capacity for emotional regulation via modulation of the parasympathetic component. Our results suggest that a sense of humor might facilitate emotional control.
\end{abstract}

Keywords: Heart Rate Variability; Sympathetic; Parasympathetic; Emotions

\section{Introduction}

Emotional perception and regulation inform the way we react to different situations, both in terms of how we feel inside and how we present ourselves to others. To form relationships and behave according to social norms we need to regulate our feelings based on ever-changing external cues. Based on these cues, we must decide quickly if our feelings are appropriate to the specific situation and adapt our behavior accordingly (Izard, Fine et al., 2001). In many conditions, such as traumatic brain injury (Thurman, Alverson et al., 1999; Bornhofen \& McDonald, 2008; McDonald, Bornhofen et al., 2009; de Sousa, McDonald et al., 2012; Hammond, Davis et al., 2012), post-traumatic stress disorder (Aupperle, Allard et al. 2012), and autism (Bal, Harden et al., 2010), this ability is impaired leading to overreaction or inappropriate behavior. A basic understanding of how healthy individuals perceive, react to, and regulate their response to various emotions is first necessary before we can begin to de-

"Corresponding author. termine how these processes may differ in those with emotional dysfunction and dysregulation.

When we feel an emotion, our subjective emotional experiences are accompanied by measurable physiological changes in heart rate, skin conductance and respiratory rate (Zuckerman, Klorman et al., 1981; Gross \& Levenson, 1993; Gross \& Levenson, 1997). These parameters measure arousal, reflecting activity of the autonomic nervous system (ANS), but do not differentiate between activity of the sympathetic and parasympathetic branches of the ANS. According to Porges' polyvagal theory, parasympathetic influence on the heart allows one to adjust metabolic output to optimize social interactions; in contrast, sympathetic activity is necessary for mobilization during threatening situations (Porges, 1995; Porges, 2007). While both branches influence heart rate, they do so by different mechanisms which are activated by specific environmental circumstances. For example, an increase in heart rate in response to a fearful stimulus may, in some cases, be a result of sympathetic activation, while in others may arise from parasympathetic 
withdrawal (Stifter, Dollar et al., 2011). Therefore, to better understand the autonomic mechanisms of emotional reactivity and regulation, it would be prudent to study activity patterns of both the sympathetic and parasympathetic branches of the ANS and examine how they interact in response to distinct affective stimuli.

Sympathetic and parasympathetic output is thought to be regulated by the brain's central autonomic network (CAN) (Benarroch, 1993), which includes regions of the cortex, brainstem and limbic systems, and is involved in a wide range of processes from homeostasis to goal-directed behavior (Appelhans \& Luecken, 2008). By integrating information about the external environment with that of the body's internal physiological state, the CAN modulates activity of the sympathetic and parasympathetic branches of the nervous system to influence heart rate. Sympathetic signals influence the sinoatrial node via the neurotransmitter norepinephrine to gradually increase heart rate, achieving peak effect after 4 seconds and returning to baseline after 20 seconds. In contrast, parasympathetic signaling via acetylcholine slows heart rate with a short response latency, achieving peak effect after 0.5 seconds and returning to baseline within 1 second (Bazhenova, Plonskaia et al., 2001; Pumprla, Howorka et al., 2002). The frequency spectrum of heart rate variability (HRV) thus reflects sympathetic and parasympathetic influences on heart rate (Appelhans \& Luecken, 2006). HRV, when analyzed concurrently with the respiratory signal calculates measures of parasympathetic activity (RFA, Respiratory Frequency Area, from higher frequencies) and sympathetic activity (LFA, Low Frequency Area, from lower frequencies) (Akselrod, Gordon et al., 1981; Aysin \& Aysin, 2006).

The goal of this study is to elucidate the autonomic mechanisms of emotional reactivity and regulation in response to ecologically-valid affective stimuli. We used film clips validated to elicit specific emotional responses (Rottenberg, Ray et al., 2007; Schaefer, Nils et al., 2010) and measured the frequency spectra of HRV and respiratory activity when participants watched the videos.

\section{Methods}

\section{Subjects}

Seven healthy subjects, ranging in age from 23 to 40 years (mean $\pm \mathrm{SD}=29.8 \pm 6.2$ years), with no history of psychiatric disease or complicating medical problems, such as uncontrolled hypertension, diabetes, neurological illness such as stroke, epilepsy, or demyelinating disease participated in the study. Four of the subjects were female [57\%].

\section{Procedure}

After obtaining informed consent, subjects were seated at a table, in front of a computer monitor, in a well-lit, 11' $\times 23^{\prime}$ room. Three electrodes, one placed below each clavicle and one on the left lower ribcage, measured electrocardiographic and respiratory signals (ANSAR Medical Technologies, 2005). First, a baseline electrocardiographic recording was obtained with no video stimulus. Subjects were instructed to look forward, relax, and breathe normally for 2 minutes.

Subjects were then told that they were going to watch a series of eight video clips (2 - 5 minutes long) (Table 1). Each subject was randomly assigned to watch one of two sets of
Table 1.

Summary of the emotion-eliciting film stimuli.

\begin{tabular}{|c|c|c|c|}
\hline & $\begin{array}{c}\text { Target } \\
\text { emotion }\end{array}$ & Movie title \& description & $\begin{array}{l}\text { Time } \\
(\mathrm{min})\end{array}$ \\
\hline \multirow{6}{*}{ 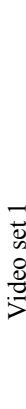 } & Neutral & $\begin{array}{l}\text { Sticks-Different colored sticks gradually } \\
\text { accumulate on a black background } \\
\text { (non-commercial screen saver, soundless). }\end{array}$ & 4.6 \\
\hline & Amusement & $\begin{array}{l}\text { Something About Mary_A man fights with a } \\
\text { small dog. }\end{array}$ & 4.33 \\
\hline & Sadness & $\begin{array}{l}\text { City of Angels-The aftermath of a woman on a } \\
\text { bike hit by a truck and dying in a man's arms. }\end{array}$ & 4.35 \\
\hline & $\begin{array}{c}\text { Sexual } \\
\text { amusement }\end{array}$ & $\begin{array}{l}\text { When Harry Met Sally-A woman loudly } \\
\text { simulates an orgasm in a crowded diner. }\end{array}$ & 3.75 \\
\hline & Fear & $\begin{array}{l}\text { The Shining-A boy plays alone in an empty } \\
\text { hallway. }\end{array}$ & 2.5 \\
\hline & & $\begin{array}{l}\text { Trainspotting-A man defecates then dives into } \\
\text { a filthy toilet. }\end{array}$ & 2.38 \\
\hline \multirow{10}{*}{ 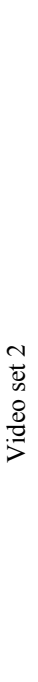 } & Disgust & $\begin{array}{l}\text { Amputation-noncommercial recording of an } \\
\text { arm amputation. }\end{array}$ & 2.3 \\
\hline & & Bad Taste-A group of young men eat vomit. & 2.96 \\
\hline & Neutral & $\begin{array}{l}\text { Sticks-Different colored sticks accumulate } \\
\text { gradually on a black background (screen saver, } \\
\text { soundless). }\end{array}$ & 4.6 \\
\hline & Amusement & $\begin{array}{l}\text { Benny and Joon-A man fools around in a } \\
\text { diner. }\end{array}$ & 3.33 \\
\hline & Sadness & $\begin{array}{l}\text { The Champ-A young boy grieves over his } \\
\text { dying father. }\end{array}$ & 3.83 \\
\hline & $\begin{array}{c}\text { Sexual } \\
\text { amusement }\end{array}$ & $\begin{array}{l}\text { A Fish Called Wanda-A woman is sexually } \\
\text { aroused while her male companion is found } \\
\text { naked by the owners of the house they are in. }\end{array}$ & 4.08 \\
\hline & Fear & $\begin{array}{l}\text { Silence of the Lambs-A woman chases a } \\
\text { dangerous man at gunpoint and comes across a } \\
\text { corpse in a bathtub. }\end{array}$ & 4.6 \\
\hline & \multirow{3}{*}{ Disgust } & Vampire's Kiss-A man eats a cockroach. & 1.65 \\
\hline & & Black Swan-A woman peels back a hangnail. & 1.87 \\
\hline & & Pink Flamingoes-A woman eats dog feces. & 2.5 \\
\hline
\end{tabular}

videos, validated to elicit the same target emotions. Each clip began with 10 seconds of written instructions, stating "Please relax and watch the + ", followed by 60 seconds of a white + sign on a black background. This was provided to bring the subjects back to baseline prior to each film-clip, which would then automatically start playing. Subjects were not informed of the sequence of the film clips. After watching each video, subjects completed a post-film questionnaire to assess the intensity of their emotional response to the film clip.

Module 1: Positive and Negative Affect. Subjects watched five short video clips targeting the following emotions: neutral, amusement, sadness, sexual amusement, and fear. The order of the clips was the same across all subjects and was chosen to counter-balance positively- and negatively-valenced affective stimuli, to prevent subjects from being overwhelmed in either direction. Before each video, subjects were told, "We will now be showing you a short film clip. It is important to us that you watch the film clip carefully."

Module 2: Disgust Regulation. Subjects watched three different video clips validated to elicit a disgust response. For the first video, subjects just watched the film clip while their reactivity was measured as in module 1 (unregulated response). For the second video, subjects were instructed to suppress their reactions. They were told, "Watch the film clip carefully. If you have any feelings as you watch the film, please try your best not to let those feelings show." For the third video, subjects were instructed to amplify their reactions. They were told, 
"Watch the film clip carefully. If you have any feelings as you watch the film clip, try your best to let those feelings show."

\section{Apparatus}

Physiological Measurements. Electrocardiographic and respiratory signals were sampled at $250 \mathrm{~Hz}$ and $50 \mathrm{~Hz}$, respectively and collected using ANSAR ANX 3.0 software (ANSAR Medical Technologies, Inc., Philadelphia, PA). Heart rate variability (HRV) was computed every 0.25 seconds and timefrequency spectral analysis was performed to quantify ANS activity. The respiratory frequency area (RFA) measured parasympathetic activity from higher frequency areas of the HRV spectrum as determined from time-frequency analyses of respiratory activity (Aysin \& Aysin, 2006). RFA represents the frequency ranges associated with Respiratory Sinus Arrhythmia, known to be a cardio-vagal response, reflecting parasympathetic activity (Akselrod, Gordon et al., 1981; Appelhans \& Luecken, 2008). Low frequency area (LFA) is defined as the area under the heart rate spectral curve over the frequency range from $0.04-0.10 \mathrm{~Hz}$, or the lower limit of RFA range (ANSAR Medical Technologies, 2005; Colombo, Shoemaker et al., 2008). By localizing and omitting the parasympathetic influence (e.g., from Respiratory Sinus Arrhythmia) from the low frequency range of HRV, LFA primarily corresponds to activity from the sympathetic nervous system (Aysin \& Aysin, 2006; Colombo, Shoemaker et al., 2008).

Self-Reported Emotional Responses. After each film clip, subjects completed a short post-film questionnaire to rate the intensity of their feelings of amusement, anger, anxiety, confusion, contempt, disgust, embarrassment, fear, guilt, happiness, interest, joy, love, pride, sadness, shame, surprise and unhappyness on a scale of 0 (none) to 8 (extreme). They were also asked to indicate if they had seen the film clip prior to the study (Rottenberg, Ray et al., 2007).

\section{Data Reduction and Statistical Analyses}

For the purposes of this study, we focused on the interval of interest (IOI) - the 30 seconds of the film clip that most strongly elicited the target emotion. Therefore, all data analysis pertains to ANSAR recordings during the IOI of each film clip. LFA and RFA values for time points at which RFA $\leq 0.1 \mathrm{bpm}^{2}$ were dropped, as they are un-interpretable for healthy subjects. For each target emotion, we measured 1) the mean LFA and RFA activity, 2) the geometric mean of the ratio between LFA and RFA (LFA/RFA) that quantifies their interrelationship, and 3) the coefficient of variability of both LFA and RFA that quantifies the fluctuation in LFA and RFA independent of the mean level.

A linear mixed effect model was used to examine how the activity levels, interrelationship and variability in LFA and RFA change across different target emotions. A random effect at subject level was used to control for individual heterogeneity. Statistical software package R 2.15.2 was used and package lmer was used to fit the model. To respect the normality assumption, a logarithm transformation was done to the geometric mean of the ratio of LFA over RFA. The results based on this particular model were then converted into multiplicative effects at the original scale. Relationships between self-reported emotional responses and autonomic parameters were examined using Spearman Rank correlations. Three levels of statistical significance $(1 \%, 5 \%$ and $10 \%)$ are reported.

\section{Results}

\section{Positive Affective Stimuli Increase LFA and Fluctuation in RFA}

Physiology. Figure 1(a) shows mean LFA and RFA values during the IOI across all subjects. Compared to neutral, mean LFA increased significantly during both amusement (mean difference $(\mathrm{md})=12.42, p<0.01)$ and sexual amusement (md $=6.08, p<0.05)$. There were no statistical differences in mean RFA across any of the stimuli compared to neutral. The mean LFA was significantly higher than the mean RFA only during amusement $(\mathrm{md}=10.25, p<0.01)$.

At the subject level, the ratio between LFA and RFA (Figure 1(b)) for the neutral stimulus was close to $1(\mathrm{LFA} / \mathrm{RFA}=0.91)$, suggesting that LFA and RFA were well balanced. For both positively-valenced stimuli, the LFA/RFA ratios were significantly higher than for the neutral or the negatively-valenced stimuli (LFA/RFA amusement $=4.84, p<0.01$; sexual amusement $=2.53, p<0.05)$, suggesting that positively-valenced stimuli elicited greater autonomic activity in the lower frequency bandwidth of HRV.

The coefficient of variation of LFA (cvLFA) was relatively constant across all affective stimuli (Figure 1(c)). Only the cvRFA for amusement was significantly higher than that for neutral ( $\mathrm{md}=0.26, p<0.01$ ). In addition, the difference between the cvRFA and cvLFA was significant during amusement $(\mathrm{md}=0.47, p<0.01)$ and fear $(\mathrm{md}=0.314, p<0.05)$. Note however that 4 out of 7 subjects had seen the fear-provoking film clip previously.

Subjective experience. To confirm that the film clips were eliciting the intended target emotion, we examined the intensity of the various emotions elicited by the film-clips on the postfilm questionnaire (Figure 1(d)). Note that the emotions that were felt most intensely for each film clip corresponded with the target emotion for that clip. Sexual amusement provoked some embarrassment while non-sexual amusement did not. There was also a strong correlation between reported embarrassment and the cvRFA $(r=0.971, p<0.01)$, suggesting that those who experienced more embarrassment showed greater fluctuation in the higher frequency component of HRV. Interestingly, there was a negative correlation between self-reported fear and both mean LFA $(r=-0.564, p=0.18)$ and mean RFA $(\mathrm{r}=-0.873, p<0.01)$.

\section{Regulation of Disgust Is Associated with Fluctuations in RFA}

Physiology. Figure 2(a) shows the mean LFA and RFA during unregulated and regulated disgust film-clips compared to neutral. The mean LFA during disgust amplification was significantly higher compared to neutral $(\mathrm{md}=13.125, p<0.01)$, unregulated disgust $(\mathrm{md}=10.366, p<0.01)$ and disgust suppression $(\mathrm{md}=10.043, p<0.01)$. The difference between mean LFA and RFA during disgust amplification was also significant ( $\mathrm{md}=18.21, p<0.05$ ). No significant differences were found in mean RFA across the stimuli. The ratio of LFA/RFA was higher during unregulated disgust $(\mathrm{LFA} / \mathrm{RFA}=2.39, p<0.10)$ and disgust amplification (LFA/RFA $=2.66, p<0.05$ ) compared to neutral and disgust suppression (Figure 2(b)). Interestingly, LFA/RFA ratios during disgust suppression ap- 

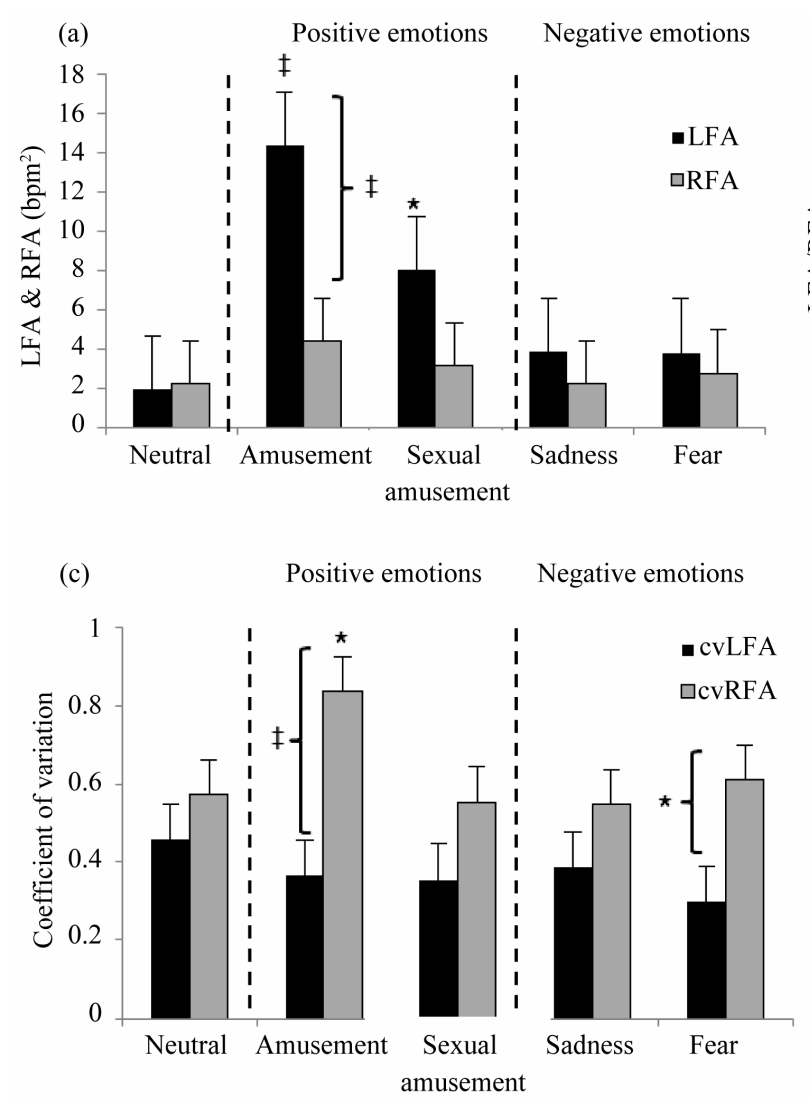

(b)

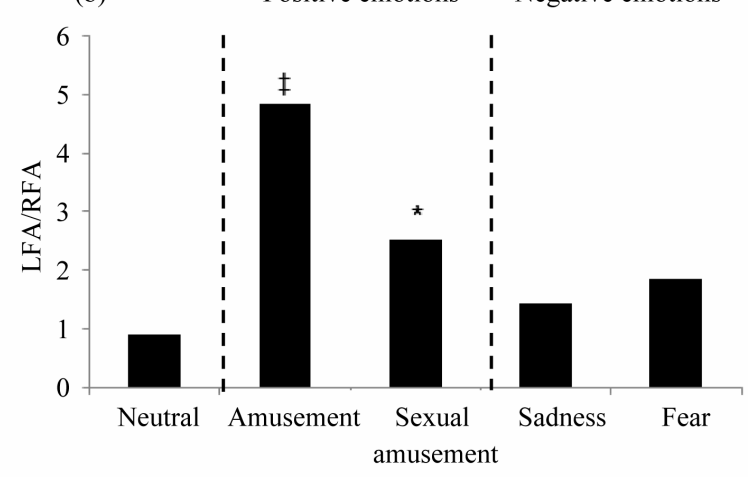

(d) Positive emotions Negative emotions

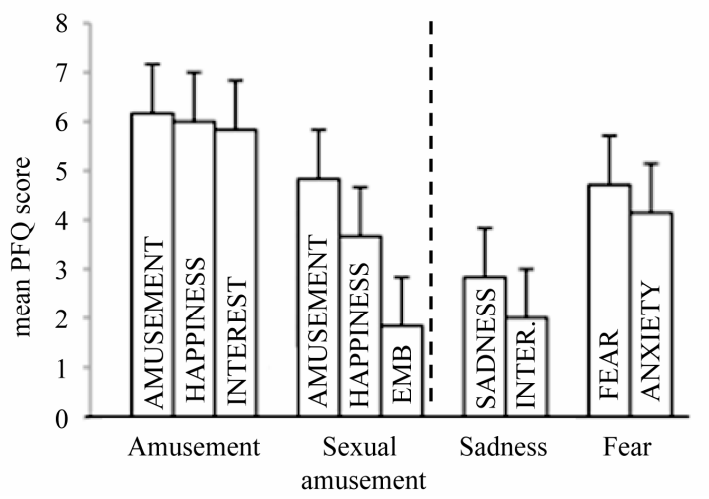

Figure 1.

Emotional reactivity in response to positively-valenced and negatively-valenced film stimuli. The symbols indicate statistical significance compared to the neutral stimulus: ${ }^{\sharp} p<0.01,{ }^{*} p<0.05$. (a) Mean LFA and RFA levels; (b) Mean ratio of LFA to RFA (LFA/RFA); (c) Coefficient of variation of LFA and RFA; (d) Self-reported emotional intensity scores for various emotions elicited by the film-clip on the post-film questionnaire (PFQ). In sexual amusement, EMB = embarrassment. In sadness, INTER = interest.

proached that of the neutral stimulus, suggesting that healthy subjects were indeed able to regulate their autonomic activity when prompted to do so. The cvRFA was significantly higher than the cvLFA during disgust suppression $(\mathrm{md}=0.31, p<$ 0.05 ) and amplification ( $\mathrm{md}=0.31, p<0.05$ ) (Figure 2(c)), suggesting that emotional regulation is reflected in increased higher frequency HRV fluctuations.

Subjective experience. Although disgust, surprise and anxiety were experienced with all three disgust-inducing film clips, the intensity of these emotions varied during suppression and amplification (Figure 2(d)). Disgust suppression was associated with less intense feelings of disgust compared with disgust amplification ( $\mathrm{md}=1.85$ units, $p<0.1$ ), but greater anxiety ( $\mathrm{md}=$ 3 units, $p<0.05$ ). Interestingly, the intensity of anxiety during disgust suppression was negatively correlated with the cvRFA ( $\mathrm{r}=-0.927, p<0.01)$, suggesting that those who experienced less anxiety showed greater fluctuation in the higher frequency component of HRV. In contrast, during amplification, anxiety was positively correlated with cvRFA $(\mathrm{r}=0.778, p<0.05)$, suggesting that those with more anxiety showed greater cvRFA, which is consistent with the direction of regulation. In addition, a strong positive correlation was noted between the intensity of amusement and cvRFA during disgust suppression $(r=0.082, p$ $<0.05$ ), suggesting that individuals who experienced higher intensity of amusement also showed greater fluctuation in the higher frequency component of HRV.

\section{Discussion}

In this study, we measured autonomic activity as reflected in the frequency spectrum of HRV, modulated by respiratory activity, when healthy subjects watched a series of film clips, which were validated to elicit specific target emotions. Our data suggest that in healthy individuals, emotional reactivity or arousal is reflected in the mean level of activity in the Low Frequency Area (LFA), a measure of sympathetic activity, whereas emotional regulation is reflected in fluctuations of the Respiratory Frequency Area (RFA), a measure of parasympathetic activity.

Both processes may occur simultaneously depending on the emotional cues, suggesting a complex interplay between sympathetic and parasympathetic mechanisms in response to changing environmental cues. Furthermore, correlations between selfreported emotional intensity and components of HRV suggest that higher positive or lower negative emotional states may increase the capacity for emotional regulation.

\section{Autonomic Basis of Emotional Reactivity}

In our study, subjects responded to positively-valenced amusing stimuli with relatively high mean LFA, and high LFA/RFA ratios compared to neutral; these emotions also evoked intense subjective feelings. Increased autonomic activity when one is amused may be due to expressive behavior such as laughing, 
(a)

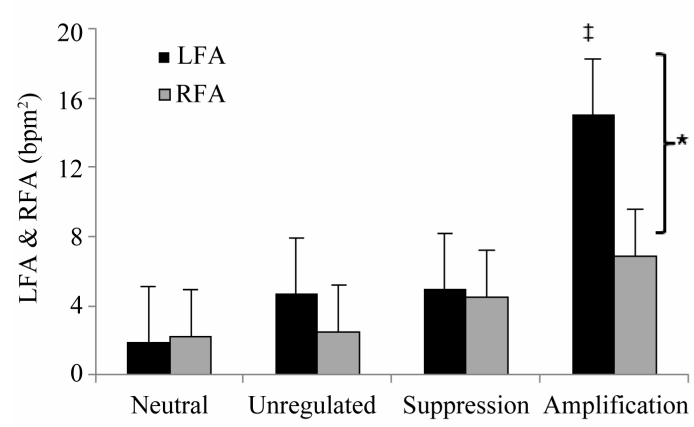

(c)

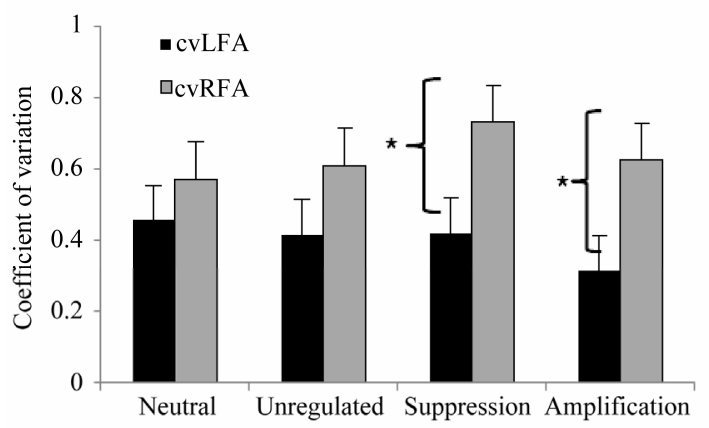

(b)

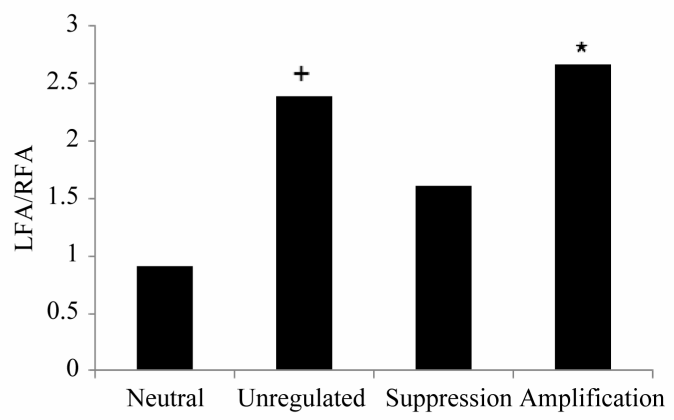

(d)

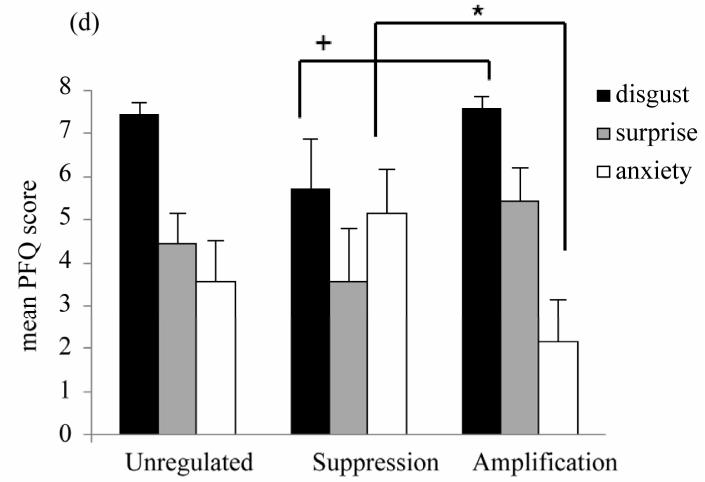

Figure 2.

Emotional reactivity and regulation in response to disgust-inducing stimuli. The symbols indicate statistical significance compared to the neutral stimulus unless indicated otherwise: ${ }^{\ddagger} p<0.01,{ }^{*} p<0.05,{ }^{+} p<0.1$. (a) Mean LFA and RFA levels; (b) Mean ratio of LFA to RFA (LFA/RFA); (c) Coefficient of variation of LFA and RFA; (d) Self-reported emotional intensity scores on the post-film questionnaire (PFQ). Note comparisons in intensity of disgust and anxiety between disgust suppression and amplification.

which has been shown to increase arousal, even after controlling for somatic activity (Sakuragi, Sugiyama et al., 2002; Giuliani, McRae et al., 2008). However, a meta-analysis of the literature on autonomic activity with induced amusement (Shiota, Neufeld et al., 2011) shows mixed results: in some studies amusement produced little or no increase in autonomic arousal relative to negative emotions (Levenson, Ekman et al., 1992), while others show reduced (Fredrickson \& Levenson, 1998; Fredrickson, Mancuso et al., 2000) or increased arousal (Neumann \& Waldstein, 2001; Mauss, Levenson et al., 2005; Giuliani, McRae et al., 2008). Some of these discrepancies may be accounted for by methodological differences. However, in our study, during the sexual amusement film clips, subjects also reported feelings of embarrassment and mild anxiety, which have been shown to decrease arousal (Gerlach, Wilhelm et al., 2003). Consequently, the increase in LFA was not as pronounced with the sexual amusement film clips as observed with the non-sexual amusement clips.

Furthermore, negatively-valenced stimuli (sadness and fear) did not elicit high LFA or LFA/RFA ratios in our study, and the subjects did not perceive the negative stimuli as intensely as the positive stimuli either. Both decreased sympathetic arousal and low LFA/RFA ratios have been noted in grief states (Sternbach, 1962; Sakuragi, Sugiyama et al., 2002), typically characterized by a flat affect. However, our results contradict other studies, which suggest that negative emotions lead to increased sympathetic activity (Fredrickson \& Levenson, 1998; Fredrickson, Mancuso et al., 2000). Some of these differences may be due to the fact that traditional HRV measures may not account for Respiratory Sinus Arrhythmia (and thus parasympathetic activity) within the low frequency range of HRV, which is subtracted out to compute the LFA with the present technology (Aysin \& Aysin, 2006). We did observe an increase in the mean LFA and high LFA/RFA ratios during disgust, particularly when subjects were asked to amplify their expression of disgust. In contrast, fear-provoking stimuli, which might be expected to produce high arousal and parasympathetic withdrawal (Berntson, Cacioppo et al., 1991), did not lead to a statistically significant difference in LFA and LFA/RFA ratio compared to the neutral stimulus. Instead, surprisingly, mean LFA was negatively correlated with the intensity of fear reported. Our unusual results may be explained by the finding that $4 / 7$ subjects had seen the fear-inducing film clip previously. It is possible that prior memory of the film and knowledge of what is going to happen next attenuated the emotional impact of the clip. Furthermore, fear elicited lower mean RFA, but higher fluctuations in RFA as seen with the amusement film clip, suggesting that parasympathetic regulatory mechanisms (see below) may have been activated. Our sample size is too small to directly relate subjective emotional intensity to autonomic reactivity. Nevertheless, taken together, our results suggest that in healthy individuals, higher intensities of subjective emotional experience, both positive (e.g., amusement) and negative (e.g., amplified disgust) elicit higher responses in the lower frequency bandwidth (LFA) of HRV. The mean LFA and LFA/RFA ratios may therefore provide an objective measure of the intensity of 
feelings.

\section{Autonomic Basis of Emotional Regulation}

In order to evaluate emotional regulation, we focused on the response to disgust-inducing stimuli. Disgust is a primal, universal emotion that may be less prone to external influences or personal preferences compared with other emotions such as amusement or sadness. In our study, subjects always started with the unregulated disgust condition, which elicited subjects' natural response to disgust. We then asked subjects to suppress their disgust; and finally, we asked them to amplify their response to disgust. We believe that this order of stimulus presentation would override any desensitization that may occur from repeated exposure to disgust-inducing stimuli. Note that three different disgust film-clips were used for the three conditions.

Unregulated disgust elicited higher mean LFA and LFA/RFA ratios compared with the neutral film clip; however, the difference only showed a trend toward significance, perhaps due to our small sample size. The self-report questionnaire indicated that disgust was strongly elicited. Previous studies have noted increased arousal in response to disgust-eliciting film stimuli (Gross \& Levenson, 1993). In contrast, when asked to suppress their disgust, subjects balanced their LFA and RFA and the LFA/RFA ratio approached that of the neutral stimulus. In addition, the intensity of disgust was reduced on the post-film questionnaire, suggesting that subjects were indeed able to suppress their feelings of disgust. The reaction to disgust was also clearly exaggerated with instructions to amplify. The mean LFA, difference between mean LFA and RFA, and LFA/RFA ratio were also higher with disgust amplification. Our data are consistent with previous studies, which found that exaggerating expressive behavior results in higher sympathetic arousal, even on non-somatic measures (Zuckerman, Klorman et al., 1981). Thus healthy subjects can clearly regulate both autonomic arousal, reflected in the mean level of the LFA, and subjective emotional responses when requested to do so.

Interestingly, both disgust suppression and amplification led to increased fluctuation in the RFA, indicating higher levels of parasympathetic activation during emotional regulation. This pattern is similar to that observed during amusement and fear film-clips, which also appeared to activate regulatory mechanisms (see above). Parasympathetic activation corresponds with a sense of calm (Fredrickson \& Levenson, 1998) and higher attention (Healy, 2010) which may be needed during regulation. We also noted a strong positive correlation between fluctuation in RFA (cvRFA) and intensity of amusement, and a strong negative correlation with anxiety during disgust suppression. These results suggest that higher positive or lower negative emotional states may increase the capacity for emotional regulation, and lend support to the theory of vagal tone as a physiologic index of stress (Porges, 1995). Interestingly, anxiety levels were greater during disgust suppression than during disgust amplification. Decreased anxiety during amplification may correspond with subjects' ability to give an unrestrained, exaggerated reaction, while increased anxiety with suppression may result from the stress of not being able to express one's feelings. Taken together, our results suggest that emotional regulation is mediated by parasympathetic activation resulting in fluctuations in the RFA, and that higher positive or lower negative emotional states may enhance the capacity for emotional regulation.

\section{Conclusion and Future Directions}

Despite the small sample size, this study leads to a number of important findings: 1) Emotional reactivity or arousal is mediated by increase in the mean level of the lower frequency component of HRV, or LFA, which measures sympathetic activity; 2) the mean LFA and LFA/RFA ratio provide an objective measure of the subjective intensity of one's feelings whether positive or negative; 3) Moment to moment fluctuations in respiratory activity modulated HRV frequency, or RFA, reflects parasympathetic-discharge-mediated emotional regulation; and 4) Positive subjective feelings appear to enhance the capacity for emotional regulation. These findings provide insight into mechanisms by which we react to and regulate our emotions to changing environmental cues, and form the basis for future research in conditions characterized by emotional dysfunction such as traumatic brain injury, post-traumatic stress disorder, and autism.

\section{Acknowledgements}

This project was funded by pilot grant from the Rusk Institute of Rehabilitation Medicine, New York University School of Medicine. We are grateful for support to IJ through the Rehabilitation Research Experience for Medical Students (RREMS) program sponsored by the Association of Academic Physiatrists and the Foundation for Physical Medicine \& Rehabilitation. We are also thankful to Shaun Nanavati for his insights in using ANSAR software, and to Dr. Joseph Colombo for the equipment loan from ANSAR.

\section{REFERENCES}

Akselrod, S., Gordon, D. et al. (1981). Power spectrum analysis of heart rate fluctuation: A quantitative probe of beat-to-beat cardiovascular control. Science, 213, 220-222. doi: $10.1126 /$ science. 6166045

ANSAR Medical Technologies (2005). The ANX version 3.0 autonomic nervous system monitor user's operations manual. ANSAR Medical Technologies, Inc., 1-20.

Appelhans, B., \& Luecken L. J. (2006). Heart rate variability as an index of regulated emotional responding. Review of General Psychology, 10, 229-240. doi:10.1037/1089-2680.10.3.229

Appelhans, B. M., \& Luecken, L. J. (2008). Heart rate variability and pain: Associations of two interrelated homeostatic processes. Biological Psychology, 77, 174-182.

doi:10.1016/i.biopsycho.2007.10.004

Aupperle, R. L., Allard, C. B. et al. (2012). Dorsolateral prefrontal cortex activation during emotional anticipation and neuropsychological performance in posttraumatic stress disorder. Archives of General Psychiatry, 69, 360-371. doi:10.1001/archgenpsychiatry.2011.1539.

Aysin, B., \& Aysin, E. (2006). Effect of respiration in heart rate variability (HRV) analysis. Conference Proceedings-IEEE Engineering in Medicine and Biology Society, 1, 1776-1779. doi:10.1109/IEMBS.2006.260773

Bal, E., Harden, E. et al. (2010). Emotion recognition in children with autism spectrum disorders: Relations to eye gaze and autonomic state. Journal of Autism and Developmental Disorders, 40, 358-370. doi:10.1007/s10803-009-0884-3

Bazhenova, O. V., Plonskaia, O. et al. (2001). Vagal reactivity and affective adjustment in infants during interaction challenges. Child Development, 72, 1314-1326. doi:10.1111/1467-8624.00350

Benarroch, E. E. (1993). The central autonomic network: Functional organization, dysfunction, and perspective. Mayo Clinic Proceedings, 68, 988-1001. doi:10.1016/S0025-6196(12)62272-1

Berntson, G. G., Cacioppo, J. T. et al. (1991). Autonomic determinism: The modes of autonomic control, the doctrine of autonomic space, 
and the laws of autonomic constraint. Psychological Review, 98, 459-487. doi:10.1037/0033-295X.98.4.459

Bornhofen, C., \& McDonald, S. (2008). Emotion perception deficits following traumatic brain injury: A review of the evidence and rationale for intervention. Journal of the International Neuropsychological Society, 14, 511-525. doi:10.1017/S1355617708080703

Colombo, J., Shoemaker, W. C. et al. (2008). Noninvasive monitoring of the autonomic nervous system and hemodynamics of patients with blunt and penetrating trauma. Journal of Trauma, 65, 1364-1373. doi:10.1097/TA.0b013e31818cc307

de Sousa, A., McDonald, S. et al. (2012). Changes in emotional empathy, affective responsivity, and behavior following severe traumatic brain injury. Journal of Clinical and Experimental Neuropsychology, 34, 606-623. doi:10.1080/13803395.2012.667067

Fredrickson, B. L., \& Levenson, R. W. (1998). Positive emotions speed recovery from the cardiovascular sequelae of negative emotions. Cognition \& Emotion, 12, 191-220. doi:10.1080/026999398379718

Fredrickson, B. L., Mancuso, R. A. et al. (2000). The undoing effect of positive emotions. Motivation and Emotion, 24, 237-258. doi:10.1023/A:1010796329158

Gerlach, A. L., Wilhelm, F. H. et al. (2003). Embarrassment and social phobia: The role of parasympathetic activation. Journal of Anxiety Disorders, 17, 197-210. doi:10.1016/S0887-6185(02)00197-4

Giuliani, N. R., McRae, K. et al. (2008). The up- and down-regulation of amusement: Experiential, behavioral, and autonomic consequences. Emotion, 8, 714-719. doi:10.1037/a0013236

Gross, J. J., \& Levenson, R. W. (1993). Emotional suppression: Physiology, self-report, and expressive behavior. Journal of Personality and Social Psychology, 64, 970-986. doi:10.1037/0022-3514.64.6.970

Gross, J. J., \& Levenson, R. W. (1997). Hiding feelings: The acute effects of inhibiting negative and positive emotion. Journal of Abnormal Psychology, 106, 95-103. doi:10.1037/0021-843X.106.1.95

Hammond, F. M., Davis, C. S. et al. (2012). Relational dimension of irritability following traumatic brain injury: A qualitative analysis. Brain Injury, 26, 1287-1296. doi:10.3109/02699052.2012.706352

Healy, B. (2010). The effect of attentional control and heart-period variability on negative affect and trait anxiety. Journal of General Psychology, 137, 140-150. doi:10.1080/00221301003645079

Izard, C., Fine, S. et al. (2001). Emotion knowledge as a predictor of social behavior and academic competence in children at risk. Psychological Science, 12, 18-23. doi:10.1111/1467-9280.00304

Levenson, R. W., Ekman, P. et al. (1992). Emotion and autonomic nervous system activity in the Minangkabau of west Sumatra. Journal of Personality and Social Psychology, 62, 972-988. doi:10.1037/0022-3514.62.6.972

Mauss, I. B., Levenson, R. W. et al. (2005). The tie that binds? Coherence among emotion experience, behavior, and physiology. Emotion, 5, 175-190. doi: $10.1037 / 1528-3542.5 \cdot 2.175$
McDonald, S., Bornhofen, C. et al. (2009). Addressing deficits in emotion recognition after severe traumatic brain injury: The role of focused attention and mimicry. Neuropsychological Rehabilitation, 19, 321-339. doi:10.1080/09602010802193989

Neumann, S. A., \& Waldstein, S. R. (2001). Similar patterns of cardiovascular response during emotional activation as a function of affective valence and arousal and gender. Journal of Psychosomatic Research, 50, 245-253. doi:10.1016/S0022-3999(01)00198-2

Porges, S. W. (1995). Cardiac vagal tone: A physiological index of stress. Neuroscience \& Biobehavioral Reviews, 19, 225-233. doi:10.1016/0149-7634(94)00066-A

Porges, S. W. (1995). Orienting in a defensive world: Mammalian modifications of our evolutionary heritage. A polyvagal theory. Psychophysiology, 32, 301-318.

doi:10.1111/j.1469-8986.1995.tb01213.x

Porges, S. W. (2007). The polyvagal perspective. Biological Psychology, 74, 116-143. doi:10.1016/j.biopsycho.2006.06.009

Pumprla, J., Howorka, K. et al. (2002). Functional assessment of heart rate variability: Physiological basis and practical applications. International Journal of Cardiology, 84, 1-14. doi:10.1016/S0167-5273(02)00057-8

Rottenberg, J., Ray, R. D. et al. (2007). Emotion elicitation using film. The handbook of motion elicitation and assessment. London: Oxford University Press.

Sakuragi, S., Sugiyama, Y. et al. (2002). Effects of laughing and weeping on mood and heart rate variability. Journal of Physiological Anthropology and Applied Human Science, 21, 159-165. doi:10.2114/jpa.21.159

Schaefer, A., Nils, F. et al. (2010). Assessing the effectiveness of a large database of emotion-eliciting films: A new tool for emotion researchers. Cognition \& Emotion, 24, 1153-1172. doi:10.1080/02699930903274322

Shiota, M. N., Neufeld, S. L. et al. (2011). Feeling good: Autonomic nervous system responding in five positive emotions. Emotion, 11, 1368-1378. doi:10.1037/a0024278

Sternbach, R. A. (1962). Assessing differential autonomic patterns in emotions. Journal of Psychosomatic Research, 6, 87-91. doi:10.1016/0022-3999(62)90059-4

Stifter, C. A., Dollar, J. M. et al. (2011). Temperament and emotion regulation: The role of autonomic nervous system reactivity. Developmental Psychobiology, 53, 266-279. doi:10.1002/dev.20519

Thurman, D. J., Alverson, C. et al. (1999). Traumatic brain injury in the United States: A public health perspective. Journal of Head Trauma Rehabilitation, 14, 602-615. doi:10.1097/00001199-199912000-00009

Zuckerman, M., Klorman, R. et al. (1981). Facial, autonomic, and subjective components of emotion: The facial feedback hypothesis versus externalizer-internalizer distinction. Journal of Personality and Social Psychology, 41, 929-944. doi:10.1037/0022-3514.41.5.929 